\title{
VERIFIABLE CONDITIONS FOR OPENNESS AND REGULARITY OF MULTIVALUED MAPPINGS IN BANACH SPACES
}

\author{
A. JOURANI AND L. THIBAULT
}

\begin{abstract}
This paper establishes verifiable conditions in terms of approximate subdifferentials implying openness and metric regularity of multivalued mappings in Banach spaces. The results are then applied to derive Lagrange multipliers for general nonsmooth vector optimization problems.
\end{abstract}

\section{INTRODUCTION}

It has been observed by Graves [13] and Ljusternik [27] that a $C^{1}$ mapping $f$ from a Banach space $X$ into a Banach space $Y$ with a surjective derivative at $x_{0}$ satisfies the distance estimate

$$
\operatorname{dist}\left(x, f^{-1}(y)\right) \leq a \operatorname{dist}(y, f(x))
$$

for all $x$ in a neighbourhood of $x_{0}$ and all $y$ in a neighbourhood of $y_{0}$. This distance estimate is actually called the metric regularity of $f$ at $x_{0}$. Another important condition ensuring the metric regularity of multivalued mappings with closed and convex graphs has been discovered by Robinson [34] and Ursescu [42]. Robinson [35] has also studied the case of the multivalued mapping $F(x)=-f(x)+P$ where $f$ is a $C^{1}$ mapping and $P$ is a closed convex cone in $Y$.

Since these works, many papers have been devoted to the metric regularity of multivalued mappings or nonsmooth mappings. The important papers by Mordukhovich [30-31] have completely solved the problem for $X$ and $Y$ finite dimensional. Indeed in [30-31], general verifiable necessary and sufficient conditions are given for any multivalued mapping between finite-dimensional Banach spaces. Such general verifiable results are not known so far in the infinite-dimensional setting. In this case most of criteria ensuring the metric regularity at a given point $x_{0}$ require the verification of the formulated conditions at all points in a neighbourhood of $x_{0}$ and, moreover, they are generally stated in a complicated way. Note that some criteria (in the nonsmooth and infinite case) are established by Aubin [1], Aubin and Frankowska [2], Borwein [3], Borwein and Zhuang [6], Dolecki [8-10], Frankowska [12], Ioffe [14-15, 18], Jourani [19-21], Jourani and Thibault [22-24], Kruger [26], Rockafellar [37], Penot [33], and references therein.

Received by the editors September 22, 1993.

1991 Mathematics Subject Classification. Primary 54C60, 46A30; Secondary 90C29.

Key words and phrases. Opennes, metric regularity, approximate subdifferential, strongly compactly Lipschitzian mappings, partially compactly epi-Lipschitzian mappings, Lagrange multipliers. 
The aim of this paper is to extend to some large classes of multivalued mappings between infinite-dimensional Banach spaces the easily verifiable sufficient criteria of Ioffe [15] and Mordukhovich [30-31]. More precisely we prove, for the multivalued mappings $F: X \rightrightarrows Y$ in these classes and $y_{0} \in F\left(x_{0}\right)$, that the condition

$$
\operatorname{Ker} D^{*} F\left(x_{0}, y_{0}\right)=\{0\}
$$

ensures the metric regularity of $F$ at $\left(x_{0}, y_{0}\right)$ (where $D^{*} F\left(x_{0}, y_{0}\right)$ is the coderivative of $F$ at $\left(x_{0}, y_{0}\right)$ (see $\left.\S 2\right)$ and $\operatorname{Ker} D^{*} F\left(x_{0}, y_{0}\right)=\left\{y^{*} \in Y^{*}\right.$ : $\left.\left.0 \in D^{*} F\left(x_{0}, y_{0}\right)\left(y^{*}\right)\right\}\right)$.

\section{Preliminaries}

Throughout the paper $X, Y$, and $Z$ will be Banach spaces and $X^{*}, Y^{*}$, and $Z^{*}$ their topological duals equipped with the weak-star topology $w^{*} .\langle\cdot, \cdot\rangle$ is the canonical pairing between two dual spaces, and $B_{X}, B_{X}^{*}, \ldots$ are the closed unit balls of $X, X^{*}, \ldots d(\cdot, S)$ is the usual distance function to the set $S$

$$
d(x, S)=\inf _{u \in S}\|x-u\| .
$$

We write $x \stackrel{f}{\rightarrow} x_{0}$ and $x \stackrel{S}{\rightarrow} x_{0}$ to express $x \rightarrow x_{0}$ with $f(x) \rightarrow f\left(x_{0}\right)$ and $x \rightarrow$ $x_{0}$ with $x \in S$, respectively. We denote by $\operatorname{Gr} F$ the graph of a multivalued mapping $F$

$$
\operatorname{Gr} F=\{(x, y): y \in F(x)\} .
$$

"dom" and "int" are the abbreviations for domain and interior. If not specified, the norm in a product of two Banach spaces is defined in a natural way by $\|(a, b)\|=\|a\|+\|b\|$.

We will use the notation of Ioffe [16-17].

Let $f: X \rightarrow \mathbb{R} \cup\{+\infty\}$ be a lower semicontinuous function in a neighbourhood of $x_{0} \in X$ with $f\left(x_{0}\right)<\infty$. The approximate subdifferential (see Ioffe [16-17]), which is an extension to the context of Banach spaces of the concept. introduced by Mordukhovich [25] for finite-dimensional spaces, is defined by

$$
\partial_{A} f\left(x_{0}\right)=\bigcap_{L \in \mathscr{F}(X)} \lim \sup _{x \stackrel{f}{\rightarrow} x_{0}} \partial^{-} f_{x+L}(x)
$$

where

$$
\begin{gathered}
\partial^{-} f(x)=\left\{x^{*} \in X^{*}:\left\langle x^{*}, h\right\rangle \leq d^{-} f(x, h), \forall h \in X\right\}, \\
d^{-} f(x, h)=\liminf _{\substack{u \rightarrow h \\
t \downarrow 0}} t^{-1}(f(x+t u)-f(x)) .
\end{gathered}
$$

Here, for $S \subset X, f_{S}$ denotes the function defined by

$$
f_{S}(x)= \begin{cases}f(x) & \text { if } x \in S \\ +\infty & \text { otherwise }\end{cases}
$$

$\mathscr{F}(X)$ is the family of all finite-dimensional subspaces of $X$; and $\limsup \partial^{-} f_{x+L}(x)=\left\{x^{*} \in X^{*}: x^{*}=w^{*}-\lim x_{i}^{*}, x_{i}^{*} \in \partial f_{x_{i}+L}\left(x_{i}\right), x_{i} \stackrel{f}{\rightarrow} x_{0}\right\}$, $\mathfrak{x}^{\rightarrow} x_{0}$

that is, the set of $w^{*}$-limits of all such nets. 
The coderivative of a multivalued mapping $F: X \rightrightarrows Y$ at a point $\left(x_{0}, y_{0}\right)$ of its graph $\mathrm{Gr} F$ is the multivalued mapping $D^{*} F\left(x_{0}, y_{0}\right): Y^{*} \rightrightarrows X^{*}$ defined by

$$
D^{*} F\left(x_{0}, y_{0}\right)\left(y^{*}\right)=\left\{x^{*}:\left(x^{*},-y^{*}\right) \in \mathbb{R}_{+} \partial_{A} d\left(x_{0}, y_{0} ; \operatorname{Gr} F\right)\right\} .
$$

In the sequel we will need the following class of mappings between Banach spaces.

Definition 2.1 [23]. A mapping $g: X \rightarrow Y$ is said to be strongly compactly Lipschitzian (s.c.L.) at a point $x_{0}$ if there exist a multivalued mapping $R$ : $X \rightrightarrows \operatorname{Comp}(Y)$, where $\operatorname{Comp}(Y)$ denotes the set of all norm compact subsets of $Y$, and a function $r: X \times X \rightarrow \mathbb{R}_{+}$satisfying:

(i) $\lim _{x \rightarrow x_{0}, h \rightarrow 0} r(x, h)=0$;

(ii) there exists $\alpha>0$ such that

$$
t^{-1}(g(x+t h)-g(x)) \in R(h)+\|h\| r(x, t h) B_{Y}
$$

for all $x \in x_{0}+\alpha B_{X}, h \in \alpha B_{X}$, and $\left.t \in\right] 0, \alpha[$;

(iii) $R(0)=\{0\}$ and $R$ is upper semicontinuous.

It can be shown (see [38]) that every strongly compactly Lipschitzian mapping is locally Lipschitz. If $Y$ is finite dimensional, the concepts coincide.

The following chain rule, involving the $A$-approximate subdifferential, has been recently stated for s.c.L. mappings by Jourani and Thibault [25]. Note that this chain rule has been stated before by Ioffe [17] for maps with compact prederivatives.

Theorem 2.2 [25]. Let $g: X \rightarrow Y$ be s.c.L. at $x_{0}$, and let $f: Y \rightarrow \mathbb{R}$ be locally Lipschitz at $g\left(x_{0}\right)$. Then $f \circ g$ is locally Lipschitz at $x_{0}$ and

$$
\partial_{A}(f \circ g)\left(x_{0}\right) \subset \bigcup_{y^{*} \in \partial_{A} f\left(g\left(x_{0}\right)\right)} \partial_{A}\left(y^{*} \circ g\right)\left(x_{0}\right) .
$$

To close this section, let us give the following result, whose proof is given in Jourani [21].

Lemma 2.3. Let $C$ be a subset of $X$ containing $x_{0}$, and let $F: X \rightrightarrows Y$ be a pseudo-Lipschitz multivalued mapping at $\left(x_{0}, y_{0}\right) \in \mathrm{Gr} F$ (that is, there exists $r>0$ and $k_{F}>0$ such that for all $x, x^{\prime} \in\left(x_{0}+r B_{X}\right), F(x) \cap\left(y_{0}+r B_{Y}\right) \subset$ $\left.F\left(x^{\prime}\right)+k_{F}\left\|x-x^{\prime}\right\| B_{Y}\right)$. Then

$$
\begin{aligned}
\partial_{A} d\left(x_{0}, y_{0}, \operatorname{Gr} F^{C}\right) & \subset \partial_{A}\left(d(\cdot, F(\cdot))+\left(k_{F}+1\right) d(\cdot, C)\right)\left(x_{0}, y_{0}\right) \\
& \subset\left(k_{F}+1\right)\left(\partial_{A}\left(d\left(y_{0}, F\left(x_{0}\right)\right)+\partial_{A} d\left(x_{0}, C\right)\right) \times\{0\}\right),
\end{aligned}
$$

where $k_{F}$ is a Lipschitz constant of $F$ at $\left(x_{0}, y_{0}\right)$ and

$$
F^{C}(x)= \begin{cases}F(x) & \text { if } x \in C \\ +\varnothing & \text { otherwise }\end{cases}
$$

\section{PARTIALly COMPACTLY EPI-LipsChITZ MUlTIVAlUed MAPPINGS: EXAMPLES AND PROPERTIES}

We start this section by recalling the following definition which is due to Borwein and Strojwas [5]. 
Definition 3.1. Let $S \subset Y$ be a set, with $y_{0} \in S . S$ is said to be compactly epi-Lipschitz at $y_{0}$ if there exist $\gamma>0$ and a \|\| -compact set $H \subset Y$ such that

$$
\left.S \cap\left(y_{0}+\gamma B_{Y}\right)+t \gamma B_{Y} \subset S-t H, \quad \text { for all } t \in\right] 0, \gamma[\text {. }
$$

Rockafellar [36] introduced the notion of "epi-Lipschitz" set. Later Borwein [4] considered an intermediate notion of reasonable boundary behavior called "epi-Lipschitz-like". He has shown that every epi-Lipschitz set is epi-Lipschitzlike and that every epi-Lipschitz-like set is compactly epi-Lipschitz. Note that in finite dimension every subset is compactly epi-Lipschitz at all its points.

Using Definition 3.1 as a point of departure, we introduce similar concepts for multivalued mappings.

Definition 3.2. We will say that a multivalued mapping $F: X \rightrightarrows Y$ is partially compactly epi-Lipschitz at $\left(x_{0}, y_{0}\right) \in \mathrm{Gr} F$ if there exist $\gamma>0$ and two \|\|$-$ compact sets $H \subset X$ and $K \subset Y$ such that for all $x \in x_{0}+\gamma B_{X}, y \in\left(y_{0}+\right.$ $\left.\left.\gamma B_{Y}\right) \cap F(x), t \in\right] 0, \gamma\left[\right.$, and $b \in \gamma B_{Y}$ there exists $(h, k) \in H \times K$ satisfying

$$
y+t(b+k) \in F(x+t h) .
$$

This is equivalent to saying that

$\left(x_{0}+\gamma B_{X}\right) \times\left(y_{0}+\gamma B_{Y}\right) \cap \mathrm{Gr} F+t\left(\{0\} \times \gamma B_{Y}\right) \subset \mathrm{Gr} F-t(H \times K)$,

for all $t \in] 0, \gamma[$.

When $H=\{0\}$ we say that $F$ is uniformly compactly epi-Lipschitz at $\left(x_{0}, y_{0}\right)$. With the above notation, this means that for all $x \in x_{0}+\gamma B_{X}$ and $\left.t \in\right] 0, \gamma[$

$$
F(x) \cap\left(y_{0}+\gamma B_{Y}\right)+t \gamma B_{Y} \subset F(x)-t K .
$$

Remarks. (1) It is obvious that $F$ is partially compact epi-Lipschitz at a point $\left(x_{0}, y_{0}\right)$ whenever $\mathrm{Gr} F$ is compactly epi-Lipschitz at this point.

(2) The converse does not hold. Indeed, let $C$ be a nonempty subset of $X$ which is not compactly epi-Lipschitz at $x_{0} \in C$. Assume that $Y$ is finite dimensional, and put $F(x)=\{0\}$ if $x \in C$ and $F(x)=\varnothing$ otherwise. One easily sees that $\operatorname{Gr} F=C \times\{0\}$ is not compactly epi-Lipschitzian at $\left(x_{0}, 0\right)$ but $F$ is partially compactly epi-Lipschitz at $\left(x_{0}, 0\right)$.

The following proposition gives us a classical example of such a class of multivalued mappings.

Proposition 3.3. Let $g: X \rightarrow Y$ be a mapping which is Lipschitz at $x_{0}$, and let $D \subset Y$ be compactly epi-Lipschitz at $g\left(x_{0}\right) \in D$. Then the multivalued mapping

$$
F(x)=-g(x)+D
$$

is uniformly compactly epi-Lipschitz at $\left(x_{0}, 0\right)$ and so it is partially compactly epi-Lipschitz at $\left(x_{0}, 0\right)$.

Proof. Since $D$ is compactly epi-Lipschitz at $g\left(x_{0}\right)$, there exist $\gamma>0$ and a \| \|-compact set $K \subset Y$ such that

$$
\left.D \cap\left(g\left(x_{0}\right)+\gamma B_{Y}\right)+t \gamma B_{Y} \subset D-t K, \quad \text { for all } t \in\right] 0, \gamma[\text {. }
$$

As $g$ is Lipschitz at $x_{0}$, there are two real numbers $k_{g}>1$ and $r>0$ such that

$$
\left\|g(x)-g\left(x_{0}\right)\right\| \leq k_{g}\left\|x-x_{0}\right\|, \quad \text { for all } x \in x_{0}+r B_{X} .
$$


We may suppose that $2 k_{g} \gamma<r$. So, let $\left.x \in x_{0}+\left(\gamma /\left(2 k_{g}\right)\right) B_{X}, t \in\right] 0, \gamma / 2[, y \in$ $F(x) \cap(\gamma / 2) B_{Y}$, and $b \in t \gamma B_{Y}$. Then, by the choice of $\gamma, y+g(x) \in D \cap$ $\left(g\left(x_{0}\right)+\gamma B_{Y}\right)$ and so $y+g(x)+b \in D-t K$.

In mathematical programming problems one often considers constraints of the form

$$
g_{i}(x) \leq 0, \quad i=1, \ldots, m, \quad g_{i}(x)=0, \quad i=m+1, \ldots, n, \text { and } x \in C,
$$

where $g_{i}: X \rightarrow \mathbb{R}$ is a function and $C$ is a subset of $X$. This can be written as $g(x) \in D$ and $x \in C$ where $g=\left(g_{1}, \ldots, g_{n}\right)$ and $D=\mathbb{R}_{-}^{m} \times\{0\} \quad\left(\mathbb{R}_{-}^{m}\right.$ being the negative orthant of $\left.\mathbb{R}^{m}\right)$, or $0 \in G(x)$ with

$$
G(x)= \begin{cases}-g(x)+D & \text { if } x \in C, \\ +\varnothing & \text { otherwise. }\end{cases}
$$

Since $D$ is compactly epi-Lipschitz at $g\left(x_{0}\right) \in D$, Proposition 3.3 implies that the multivalued mapping $-g(x)+D$ is uniformly compactly epi-Lipschitz at $\left(x_{0}, 0\right)$. We easily show that $G$ is also uniformly compactly epi-Lipschitz at $\left(x_{0}, 0\right)$. More generally we have

Proposition 3.4. Let $F: X \rightrightarrows Y$ be a multivalued mapping which is uniformly compactly epi-Lipschitz at $\left(x_{0}, y_{0}\right) \in \mathrm{Gr} F$, and let $C$ be a subset of $X$ containing $x_{0}$. Then the multivalued mapping

$$
F^{C}(x)= \begin{cases}F(x) & \text { if } x \in C, \\ +\varnothing & \text { otherwise }\end{cases}
$$

is uniformly compactly epi-Lipschitz at $\left(x_{0}, y_{0}\right)$.

In the following proposition we establish the following important property of partially compactly epi-Lipschitz multivalued mappings.

Proposition 3.5. Let $F: X \rightrightarrows Y$ be a multivalued mapping which is partially compactly epi-Lipschitz at $\left(x_{0}, y_{0}\right) \in \mathrm{Gr} F$. Then, for $H$ and $K$ given by Definition 3.2, there are neighbourhoods $V$ and $W$ of $x_{0}$ and $y_{0}$ respectively and a real number $\gamma>0$ such that for each $\varepsilon \in] 0,1]$ there exist vectors $h_{1}, \ldots, h_{m} \in H$ and $k_{1}, \ldots, k_{m} \in K$ satisfying

$$
\varepsilon\left\|x^{*}\right\|+\left\|y^{*}\right\| \leq 3 \varepsilon+\gamma \max _{i=1, \ldots, m}\left|\left\langle x^{*}, h_{i}\right\rangle\right|+\gamma \max _{i=1, \ldots, m}\left|\left\langle y^{*}, k_{i}\right\rangle\right|
$$

for all $x \in V, y \in W$, and $\left(x^{*}, y^{*}\right) \in \partial_{A} d(x, y ; \mathrm{Gr} F)$.

Proof. Let $r \in] 0,1]$ be such that for all $t \in] 0, r[$

$$
\left(x_{0}+4 r B_{X}\right) \times\left(y_{0}+4 r B_{Y}\right) \cap \mathrm{Gr} F+t\left(\{0\} \times r B_{Y}\right) \subset \mathrm{Gr} F-t(H \times K) .
$$

Choose open neighbourhoods $V$ and $W$ of $x_{0}$ and $y_{0}$, respectively, such that $V \subset x_{0}+r B_{X}$ and $W \subset y_{0}+r B_{Y}$ and such that for all $(x, y) \in V \times W$, $d(x, y ; \operatorname{Gr} F)<r$. Let $\varepsilon \in] 0,1]$. Choose $h_{1}, \ldots, h_{m} \in H$ and $k_{1}, \ldots, k_{m} \in$ $K$ such that

$$
H \subset \bigcup_{i=1}^{m}\left(h_{i}+\varepsilon r^{2} B_{X}\right) \text { and } K \subset \bigcup_{i=1}^{m}\left(k_{i}+\varepsilon r^{2} B_{Y}\right)
$$

For all $x \in V, y \in W, u \in \varepsilon r B_{X}$, and $\left.t \in\right] 0, r[$ with $x+t u \in V$ there exists $(p(x, y, u, t), q(x, y, u, t)) \in \mathrm{Gr} F$ such that

(2) $\|p(x, y, u, t)-(x+t u)\|+\|q(x, y, u, t)-y\| \leq d(x+t u, y$; GrF $F)+t^{2}$ 
and hence $p(x, y, u, t) \in x_{0}+4 r B_{X}$ and $q(x, y, u, t) \in y_{0}+4 r B_{Y}$. Let $\left(x^{*}, y^{*}\right) \in \partial_{A} d(x, y ; \operatorname{Gr} F)$. Fix $u \in \operatorname{er} B_{X}, L \in \mathscr{F}(X)$ with $\left\{u, h_{1}, \ldots\right.$, $\left.h_{m}\right\} \subset L, b \in r B_{Y}$, and $M \in \mathscr{F}(Y)$ with $\left\{b, k_{1}, \ldots, k_{m}\right\} \subset M$. By the definition of approximate subdifferential we have

$$
x^{*}=w^{*}-\lim x_{j}^{*} \quad \text { and } \quad y^{*}=w^{*}-\lim y_{j}^{*}
$$

with $\left(x_{j}^{*}, y_{j}^{*}\right) \in \partial^{-} d_{\left(x_{j}, y_{j}\right)+L \times M}(\cdot, \cdot ; \operatorname{Gr} F)\left(x_{j}, y_{j}\right)$ and $\left(x_{j}, y_{j}\right) \rightarrow(x, y)$.

Choose $t_{n} \downarrow 0, j_{0}$, and $n_{0} \in \mathbb{N}$ such that for all $j \geq j_{0}$ and $n \geq n_{0}$ we have $y_{j} \in W, x_{j} \in V$, and $x_{j}+t_{n} u \in V$. For $j \geq j_{0}$ and $n \geq n_{0}$ take by (1) $h_{\alpha(n, j)} \in\left\{h_{1}, \ldots, h_{m}\right\}, k_{\beta(n, j)} \in\left\{k_{1}, \ldots, k_{m}\right\}, b_{n, j} \in B_{X}$, and $b_{n, j}^{\prime} \in B_{Y}$ satisfying

$$
\begin{aligned}
& \left(p\left(x_{j}, y_{j}, u, t_{n}\right), q\left(x_{j}, y_{j}, u, t_{n}\right)\right) \\
& \quad+t_{n}\left(h_{\alpha(n, j)}+\varepsilon r^{2} b_{n, j}, b+k_{\beta(n, j)}+\varepsilon r^{2} b_{n, j}^{\prime}\right) \in \operatorname{Gr} F .
\end{aligned}
$$

Extracting subnets if necessary we may suppose that $h_{\alpha(n, j)}=h_{l}$ and $k_{\beta(n, j)}=$ $k_{s}$ for all $j \geq j_{0}$ and $n \geq n_{0}$, where $l, s \in\{1, \ldots, m\}$. So for $p_{n, j}:=$ $p\left(x_{j}, y_{j}, u, t_{n}\right)$ and $q_{n, j}:=q\left(x_{j}, y_{j}, u, t_{n}\right)$ we have by (2) and (3)

$$
\begin{aligned}
t_{n}^{-1}( & \left.\left(x_{j}+t_{n}\left(u+h_{l}\right), y_{j}+t_{n}\left(b+k_{s}\right) ; \mathrm{Gr} F\right)-d\left(x_{j}, y_{j} ; \mathrm{Gr} F\right)\right) \\
\leq & t_{n}^{-1}\left(d\left(p_{n, j}+t_{n} h_{l}, q_{n, j}+t_{n}\left(b+k_{s}\right) ; \mathrm{Gr} F\right)-d\left(x_{j}, y_{j} ; \mathrm{Gr} F\right)\right) \\
& +t_{n}^{-1}\left(\left\|x_{j}+t_{n} u-p_{n, j}\right\|+\left\|y_{j}-q_{n, j}\right\|\right) \\
\leq & 2 \varepsilon r^{2}+t_{n}^{-1}\left(d\left(x_{j}+t_{n} u, y_{j} ; \mathrm{Gr} F\right)-d\left(x_{j}, y_{j} ; \mathrm{Gr} F\right)\right)+t_{n} \\
\leq & 2 \varepsilon r^{2}+\varepsilon r+t_{n} \\
\leq & 3 \varepsilon r+t_{n} .
\end{aligned}
$$

Setting $g:=d(\cdot, \cdot, \operatorname{Gr} F)$ we get

$$
d^{-} g_{\left(x_{j}, y_{j}\right)+L \times M}\left(x_{j}, y_{j} ; u+h_{l}, b+k_{s}\right) \leq 3 \varepsilon r,
$$

and hence

$$
\left\langle x_{j}^{*}, u\right\rangle+\left\langle y_{j}^{*}, b\right\rangle \leq 3 \varepsilon r+\max _{i=1, \ldots, m}\left|\left\langle x_{j}^{*}, h_{i}\right\rangle\right|+\max _{i=1, \ldots, m}\left|\left\langle y_{j}^{*}, k_{i}\right\rangle\right| .
$$

It follows that

$$
\left\langle x^{*}, u\right\rangle+\left\langle y^{*}, b\right\rangle \leq 3 \varepsilon r+\max _{i=1, \ldots, m}\left|\left\langle x^{*},,_{i}\right\rangle\right|+\max _{i=1, \ldots, m}\left|\left\langle y^{*}, k_{i}\right\rangle\right|,
$$

and hence we may conclude that

$$
\varepsilon\left\|x^{*}\right\|+\left\|y^{*}\right\| \leq 3 \varepsilon+r^{-1} \max _{i=1, \ldots, m}\left|\left\langle x^{*}, h_{i}\right\rangle\right|+r^{-1} \max _{i=1, \ldots, m}\left|\left\langle y^{*}, k_{i}\right\rangle\right| .
$$

As a consequence of this proposition we have

Corollary 3.6. Let $F: X \rightrightarrows Y$ be a multivalued mapping which is uniformly compactly epi-Lipschitz at $\left(x_{0}, y_{0}\right) \in \mathrm{Gr} F$. Then, for $K$ given by Definition 3.2 , there are neighbourhoods $V$ and $W$ of $x_{0}$ and $y_{0}$, respectively, and a real number $\gamma>0$ such that for each $\varepsilon \in] 0,1\left[\right.$ there exist vectors $k_{1}, \ldots, k_{m} \in K$ satisfying

$$
\varepsilon\left\|x^{*}\right\|+\left\|y^{*}\right\| \leq 3 \varepsilon+\gamma \max _{i=1, \ldots, m}\left|\left\langle y^{*}, k_{i}\right\rangle\right|
$$

for all $x \in V, y \in W$, and $\left(x^{*}, y^{*}\right) \in \partial_{A} d(x, y ; \operatorname{Gr} F)$. 
In particular if $D \subset Y$ is compactly epi-Lipschitz at $y_{0} \in D$ one gets, by setting $F(x)=D$, for all $x \in X$, that

$$
\left\|y^{*}\right\| \leq 3 \varepsilon+\gamma \max _{i=1, \ldots, m}\left|\left\langle y^{*}, k_{i}\right\rangle\right|
$$

for all $y \in V$ and $y^{*} \in \partial_{A} d(y, D)$.

Remarks. (1) From the second part of this corollary we may say, in particular, that in $\partial_{A} d(y, D)$, weak-star and strong convergences to zero are equivalent, that is,

$$
y_{j}^{*} \stackrel{w^{*}}{\rightarrow} 0 \quad \text { iff }\left\|y_{j}^{*}\right\| \rightarrow 0 .
$$

(2) Loewen [28] has shown that in weakly locally compact cones (of reflexive Banach spaces), weak-star and strong convergences of sequences to zero are equivalent.

\section{Metric Regularity}

The following notion of metric regularity is now recognized to be a very important tool in nonsmooth analysis and in optimization theory.

Definition 4.1 [34-35]. One says that a multivalued mapping $F: X \rightrightarrows Y$ is metrically regular at $\left(x_{0}, y_{0}\right) \in \mathrm{Gr} F$ if there exist two real numbers $a \geq 0$ and $r>0$ such that

$$
d\left(x, F^{-1}(y)\right) \leq \operatorname{ad}(y, F(x))
$$

for all $x \in x_{0}+r B_{X}$ and $y \in y_{0}+r B_{Y}$ with $d(y, F(x)) \leq r$, where $F^{-1}(y)=$ $\{x \in X: y \in F(x)\}$. Here we adopt the convention $d(x, \varnothing)=+\infty$.

Before giving our main result of this section, we recall the following important lemma whose proof is given in Jourani [19]. Let us note that its proof is along the lines of those given by Borwein and Zhuang [6], Borwein [3], and Ioffe [14].

Lemma 4.2. Let $F: X \rightrightarrows Y$ be a multivalued mapping of closed graph, and let $\left(x_{0}, y_{0}\right) \in \mathrm{Gr} F$. If $F$ is not metrically regular at $\left(x_{0}, y_{0}\right)$, then there are $s_{n} \downarrow 0$ $\left(s_{n}<1\right), x_{n} \rightarrow x_{0}, z_{n} \rightarrow y_{0}$, and $y_{n} \rightarrow y_{0}$ such that for all positive integer $n$

$$
\left(x_{n}, z_{n}\right) \in \mathrm{Gr} F, \quad y_{n} \notin F\left(x_{n}\right),
$$

and

for all $(x, y) \in \mathrm{Gr} F$.

$$
\left\|z_{n}-y_{n}\right\| \leq\left\|y-y_{n}\right\|+s_{n}\left(\left\|x-x_{n}\right\|+\left\|y-z_{n}\right\|\right)
$$

Theorem 4.3. Let $F: X \rightrightarrows Y$ be a multivalued mapping whose graph is closed. Suppose that $F$ is partially compactly epi-Lipschitz at $\left(x_{0}, y_{0}\right) \in \mathrm{Gr} F$. Suppose also that for all nonzero $y^{*} \in Y^{*}$

$$
\operatorname{Ker} D^{*} F\left(x_{0}, y_{0}\right)=\{0\} .
$$

Then $F$ is metrically regular at $\left(x_{0}, y_{0}\right)$.

Proof. Suppose the contrary. Then, by Lemma 4.2, there are $s_{n} \downarrow 0 \quad\left(s_{n}<1\right)$, $x_{n} \rightarrow x_{0}, z_{n} \rightarrow y_{0}$, and $y_{n} \rightarrow y_{0}$ such that for all $n$

$$
\left(x_{n}, z_{n}\right) \in \mathrm{Gr} F, \quad y_{n} \notin F\left(x_{n}\right),
$$

and

$$
\left\|z_{n}-y_{n}\right\| \leq\left\|y-y_{n}\right\|+s_{n}\left(\left\|x-x_{n}\right\|+\left\|y-z_{n}\right\|\right)
$$


for all $(x, y) \in \operatorname{Gr} F$. Proposition 2.4.3 in Clarke [7] implies that $\left(x_{n}, z_{n}\right)$ is a local minimum of the function

$$
f_{n}(x, y)=\left\|y-y_{n}\right\|+2 d(x, y ; \operatorname{Gr} F)+s_{n}\left(\left\|x-x_{n}\right\|+\left\|y-z_{n}\right\|\right) .
$$

So $(0,0) \in \partial_{A} f_{n}\left(x_{n}, z_{n}\right)$ and hence, by subdifferential calculus rules (see Ioffe [16-17]),

$$
(0,0) \in\{0\} \times S\left(y_{n}, z_{n}\right)+2 \partial_{A} d\left(x_{n}, z_{n} ; \mathrm{Gr} F\right)+s_{n}\left(B_{X}^{*} \times B_{Y}^{*}\right),
$$

where $S\left(y_{n}, z_{n}\right)=\left\{y^{*} \in Y^{*}:\left\|y^{*}\right\|=1\right.$ and $\left.\left\langle y^{*}, z_{n}-y_{n}\right\rangle=\left\|z_{n}-y_{n}\right\|\right\}$. So there are $y_{n}^{*} \in S\left(y_{n}, z_{n}\right)$ and $\left(x_{n}^{*}, z_{n}^{*}\right) \in 2 \partial_{A} d\left(x_{n}, z_{n} ; \mathrm{Gr} F\right)$ such that

$$
\left\|y_{n}^{*}+z_{n}^{*}\right\| \leq s_{n}
$$

and

$$
\left\|x_{n}^{*}\right\| \leq s_{n} .
$$

As the sequences $\left(x_{n}^{*}\right),\left(y_{n}^{*}\right)$, and $\left(z_{n}^{*}\right)$ are bounded, extracting subnets if necessary, we may assume that $x_{n}^{*} \stackrel{w^{*}}{\rightarrow} 0, z_{n}^{*} \stackrel{w^{*}}{\rightarrow}-y^{*}$, and $y_{n}^{*} \stackrel{w^{*}}{\rightarrow} y^{*}$. By the upper semicontinuity property of the approximate subdifferential we get

$$
\left(0,-y^{*}\right) \in 2 \partial_{A} d\left(x_{0}, y_{0} ; \mathrm{Gr} F\right) .
$$

The theorem will therefore be proved if we show that $y^{*} \neq 0$ because we will arrive at a contradiction with (RC). Now, using Proposition 3.5 we get the existence of $\gamma>0$ such that for all $\varepsilon \in] 0,1]$ there exist $h_{1}, \ldots, h_{m} \in X$ and $k_{1}, \ldots, k_{m} \in Y \quad\left(h_{1}, \ldots, h_{m}, k_{1}, \ldots, k_{m}\right.$ not depending on $\left.n\right)$ satisfying

$$
\varepsilon\left\|x_{n}^{*}\right\|+\left\|z_{n}^{*}\right\| \leq 6 \varepsilon+\gamma \max _{i=1, \ldots, m}\left|\left\langle x_{n}^{*}, h_{i}\right\rangle\right|+\gamma \max _{i=1, \ldots, m}\left|\left\langle z_{n}^{*}, k_{i}\right\rangle\right|
$$

and hence

$$
\varepsilon\left\|x_{n}^{*}\right\|+\left\|y_{n}^{*}\right\|-\left\|y_{n}^{*}+z_{n}^{*}\right\| \leq 6 \varepsilon+\gamma \max _{i=1, \ldots, m}\left|\left\langle x_{n}^{*}, h_{i}\right\rangle\right|+\gamma \max _{i=1, \ldots, m}\left|\left\langle z_{n}^{*}, k_{i}\right\rangle\right| .
$$

Using (4) we have at the limit

$$
1 \leq 6 \varepsilon+\gamma \max _{i=1, \ldots, m}\left|\left\langle y^{*}, k_{i}\right\rangle\right|,
$$

which ensures that $y^{*} \neq 0$ and the proof is complete.

As a consequence of Theorem 4.3 we immediately have the following result concerning the openness of multivalued mappings.

Corollary 4.4. Let $F$ be as in Theorem 4.3. Then, under the assumptions of this theorem, the multivalued mapping $F$ is open at a linear rate at $\left(x_{0}, y_{0}\right)$; that is, there are neighbourhoods $V$ and $W$ of $x_{0}$ and $y_{0}$, respectively, and two real numbers $s>0$ and $b>0$ such that

$$
y+t B_{Y} \subset F\left(x+t b B_{X}\right)
$$

for all $(x, y) \in V \times W \cap \mathrm{Gr} F$ and $t \in] 0, s[$.

Proof. By Theorem 4.3 there exist $a>0$ and $r>0$ such that

$$
d\left(x, F^{-1}\left(y^{\prime}\right)\right) \leq \operatorname{ad}\left(y^{\prime}, F(x)\right)
$$

for all $x \in x_{0}+r B_{X}$ and $y^{\prime} \in y_{0}+r B_{Y}$ with $d\left(y^{\prime}, F(x)\right) \leq r$. So let $x \in$ $\left.x_{0}+r B_{X}, y \in\left(y_{0}+(r / 2) B_{Y}\right) \cap F(x), t \in\right] 0, r / 2\left[\right.$, and $y^{\prime} \in y+t B_{Y}$. Then 
$y^{\prime} \in y_{0}+r B_{Y}$ with $d\left(y^{\prime}, F(x)\right) \leq r$ and so, by (5), there exists $x^{\prime} \in F^{-1}\left(y^{\prime}\right)$ such that

$$
\left\|x-x^{\prime}\right\| \leq 2 a\left\|y-y^{\prime}\right\| \leq 2 a t
$$

and hence $y^{\prime} \in F\left(x^{\prime}\right) \subset F\left(x+2 a t B_{X}\right)$.

Corollary 4.5. Let $F: X \rightrightarrows Y$ be a multivalued mapping whose graph is closed, and let $C$ be a closed subset of $X$ containing $x_{0}$. Suppose that $F$ is uniformly compactly epi-Lipschitz and pseudo-Lipschitz at $\left(x_{0}, y_{0}\right) \in \mathrm{Gr} F$. Suppose also that for all nonzero $y^{*} \in Y^{*}$

$$
0 \notin D^{*} F\left(x_{0}, y_{0}\right)\left(y^{*}\right)+\mathbb{R}_{+} \partial_{A} d\left(x_{0}, C\right) .
$$

Then the multivalued mapping $F^{C}: X \rightrightarrows Y$ as defined above is metrically regular at $\left(x_{0}, y_{0}\right)$.

Proof. It suffices to show that the regularity condition (RC) of Theorem 4.3 holds for $F^{C}$. Indeed, let $\left(0, y^{*}\right) \in \partial_{A} d\left(x_{0}, y_{0} ; \mathrm{Gr} F^{C}\right)$. By Lemma 2.3, there exists $k>0$ such that

$$
\partial_{A} d\left(x_{0}, y_{0} ; \operatorname{Gr} F^{C}\right) \subset k\left[\partial_{A} d\left(x_{0}, y_{0} ; \mathrm{Gr} F\right)+\partial_{A} d\left(x_{0}, C\right) \times\{0\}\right] .
$$

Therefore

$$
0 \in D^{*} F\left(x_{0}, y_{0}\right)\left(y^{*}\right)+\mathbb{R}_{+} \partial_{A} d\left(x_{0}, C\right),
$$

which implies by assumptions that $y^{*}=0$. So Proposition 3.4 and Theorem 4.3 complete the proof.

\section{APPLICATION TO VECTOR OPTIMIZATION PROBLEMS}

In this section, we consider Lagrange multipliers for vector optimization problems of the form

$$
\begin{array}{ll}
\text { minimize } & f(x) \\
\text { subject to } & x \in C, \\
& 0 \in F(x),
\end{array}
$$

where $f: X \rightarrow Z$ is a mapping, $Z$ is a Banach space, $F: X \rightrightarrows Y$ is a multivalued mapping, and $C$ is a subset of $X$.

Let $P$ be a closed convex cone of $Z$ with nonempty interior and such that $P \cap(-P)=\{0\}$.

Recall that a point $x_{0} \in C \cap F^{-1}(0)$ is a weak Pareto local minimum for the problem $(\mathrm{P})$ if there exists a neighbourhood $V$ of $x_{0}$ such that for all $x \in V \cap C \cap F^{-1}(0)$, with $x \neq x_{0}$,

$$
f\left(x_{0}\right)-f(x) \notin \operatorname{int} P \text {. }
$$

In the sequel we assume that $f$ is strongly compactly Lipschitzian at $x_{0} \in$ $C \cap F^{-1}(0)$ and $C$ is closed.

We start this section by recalling the following lemma whose proof is given in Thibault [40].

Lemma 5.1. Assume that $x_{0}$ is a weak Pareto local minimum for the problem (P). Then for each element $b$ in $f\left(x_{0}\right)$-int $P$ there exists a continuous seminorm $p$ on $Z$ which is monotonically increasing on $P$ and such that

$$
1=p\left(f\left(x_{0}\right)-b\right) \leq p(f(x)-b)
$$

for all $x \in C \cap F^{-1}(0)$ in some neighbourhood of $x_{0}$. 
Before stating our main result of this section, let us establish the following lemma which is in the line of Proposition 2.7 in Thibault [41].

Lemma 5.2. Let $F: X \rightrightarrows Y$ be a multivalued mapping which is pseudo-Lipschitz at $\left(x_{0}, y_{0}\right) \in \mathrm{Gr} F$. Then there exists $k>0$ such that

$$
\begin{aligned}
\partial_{A} d\left(y_{0}, F(\cdot)\right)\left(x_{0}\right) & \subset \bigcup_{y^{*} \in B_{Y}^{*}}\left\{x^{*} \in X^{*}:\left(x^{*}, y^{*}\right) \in k \partial_{A} d\left(x_{0}, y_{0} ; \mathrm{Gr} F\right)\right\} \\
& \subset \bigcup_{y^{*} \in B_{Y}^{*}}\left\{x^{*} \in X^{*}:\left(x^{*}, y^{*}\right) \in k \partial_{A} d\left(y_{0} ; F\left(x_{0}\right)\right)\right\} .
\end{aligned}
$$

Proof. Following Rockafellar [37], $F$ is pseudo-Lipschitz at $\left(x_{0}, y_{0}\right)$ iff the function $(x, y) \rightarrow d(y, F(x))$ is locally Lipschitz at $\left(x_{0}, y_{0}\right)$. Let $k_{F}$ be a Lipschitz constant of this function at $\left(x_{0}, y_{0}\right)$. Thus (see Ioffe [17])

$$
\partial_{A} d\left(y_{0}, F(\cdot)\right)\left(x_{0}\right)=\bigcap_{L \in \mathscr{F}(X)} \limsup _{\substack{x \rightarrow x_{0} \\ \varepsilon \downarrow 0}}\left[\partial_{\varepsilon}^{-} d_{x+L}\left(y_{0}, F(\cdot)\right)(x) \cap\left(k_{F}+\varepsilon\right) B_{X}^{*}\right],
$$

where for a function $f: X \rightarrow \mathbb{R} \cup\{+\infty\}$, with $f(x)<\infty$, the set $\partial_{\varepsilon}^{-} f(x)$ is given by $\partial_{\varepsilon}^{-} f(x)=\left\{x^{*} \in X^{*}:\left\langle x^{*}, h\right\rangle \leq d^{-} f(x, h)+\varepsilon\|h\|, \forall h \in X\right\}$. So for $x^{*} \in \partial_{A} d\left(y_{0}, F(\cdot)\right)\left(x_{0}\right)$ and $L \in \mathscr{F}(X)$ there exist nets $x_{i} \rightarrow x_{0}, x_{i}^{*} \stackrel{w^{*}}{\rightarrow} x^{*}$, and $\varepsilon_{i} \downarrow 0 \quad\left(\varepsilon_{i}<1\right)$ such that

$$
x_{i}^{*} \in \partial_{\varepsilon_{i}}^{-} d_{x_{i}+L}\left(y_{0}, F(\cdot)\right)\left(x_{i}\right) \cap\left(k_{F}+1\right) B_{X}^{*} .
$$

Using Lemma 1 in Ioffe [15] and Proposition 2.4.3 in Clarke [7] we get for all $\varepsilon \in] 0,1[$ that the function

(6) $x \rightarrow d\left(y_{0}, F(x)\right)-\left\langle x_{i}^{*}, x-x_{i}\right\rangle+\left(\varepsilon+\varepsilon_{i}\right)\left\|x-x_{i}\right\|+\left(2 k_{F}+2\right) d\left(x, x_{i}+L\right)$

attains a local minimum at $x_{i}$. Choose $y_{i} \in F\left(x_{i}\right)$ such that

$$
\left\|y_{0}-y_{i}\right\| \leq d\left(y_{0}, F\left(x_{i}\right)\right)+\varepsilon^{2} \text {. }
$$

Using (6) and (7) we obtain

$\varepsilon^{2}+\left\|y_{0}-y\right\|-\left\langle x_{i}^{*}, x-x_{i}\right\rangle+\left(\varepsilon+\varepsilon_{i}\right)\left\|x-x_{i}\right\|+\left(2 k_{F}+2\right) d\left(x, x_{i}+L\right) \geq\left\|y_{0}-y_{i}\right\|$ for all $(x, y) \in \operatorname{Gr} F \cap\left(x_{i}+r_{i} B_{X}\right) \times\left(y_{i}+r_{i} B_{Y}\right)$ for some real number $r_{i}>0$. Set $f_{i}(x, y)=\left\|y_{0}-y\right\|-\left\langle x_{i}^{*}, x-x_{i}\right\rangle+\left(\varepsilon+\varepsilon_{i}\right)\left\|x-x_{i}\right\|+\left(2 k_{F}+2\right) d\left(x, x_{i}+L\right)$ and $E=\operatorname{Gr} F \cap\left(x_{i}+r_{i} B_{X}\right) \times\left(y_{i}+r_{i} B_{Y}\right)$. Then

$$
f_{i}\left(x_{i}, y_{i}\right) \leq \inf _{(x, y) \in E} f_{i}(x, y)+\varepsilon^{2} .
$$

Applying the Ekeland variational principle [11] to $f_{i}$ on $E$ we have the existence of $\left(x_{\varepsilon, i}, y_{\varepsilon, i}\right)$ in $E$ satisfying

$$
\left\|x_{\varepsilon, i}-x_{i}\right\|+\left\|y_{\varepsilon, i}-y_{i}\right\| \leq \varepsilon
$$

and

$$
f_{i}\left(x_{\varepsilon, i}, y_{\varepsilon, i}\right) \leq f_{i}(x, y)+\varepsilon\left(\left\|x_{\varepsilon, i}-x\right\|+\left\|y_{\varepsilon, i}-y\right\|\right), \quad \text { for all }(x, y) \in E .
$$

Then, by Proposition 2.4.3 in Clarke [7], $\left(x_{\varepsilon, i}, y_{\varepsilon, i}\right)$ is a local minimum of the function

$$
(x, y) \rightarrow f_{i}(x, y)+\varepsilon\left(\left\|x_{\varepsilon, i}-x\right\|+\left\|y_{\varepsilon, i}-y\right\|\right)+3\left(k_{F}+1\right) d(x, y ; E) .
$$


So, by subdifferential calculus rules (see Ioffe [16-17])

$$
\begin{aligned}
\left(x_{i}^{*}, 0\right) \in\{0\} \times B_{Y}^{*} & +3\left(k_{F}+1\right) \partial_{A} d\left(x_{\varepsilon, i}, y_{\varepsilon, i} ; \mathrm{Gr} F\right) \\
& +\left(2 \varepsilon+\varepsilon_{i}\right) B_{X}^{*} \times B_{Y}^{*}+2\left(k_{F}+1\right) \partial_{A} d\left(x_{\varepsilon, i}, x_{i}+L\right) \times\{0\}
\end{aligned}
$$

because $\partial_{A} d\left(x_{\varepsilon, i}, y_{\varepsilon, i} ; \mathrm{Gr} F\right)=\partial_{A} d\left(x_{\varepsilon, i}, y_{\varepsilon, i} ; E\right)$. By (7) and (8) we have $x_{\varepsilon, i} \rightarrow x_{0}$ and $y_{\varepsilon, i} \rightarrow y_{0}$. So the upper semicontinuity of the approximate subdifferential and the weak-star compactness of the sets $\partial_{A} d\left(x_{\varepsilon, i}, y_{\varepsilon, i} ; \mathrm{Gr} F\right)$, $B_{X}^{*}, B_{Y}^{*}$, and $\partial_{A} d\left(x_{\varepsilon, i}, x_{i}+L\right)$ imply

$$
\left(x^{*}, 0\right) \in\{0\} \times B_{Y}^{*}+3\left(k_{F}+1\right) \partial_{A} d\left(x_{0}, y_{0} ; \mathrm{Gr} F\right)+L^{\perp} \times\{0\}
$$

for all $L \in \mathscr{F}(X)$, where $L^{\perp}=\left\{x^{*} \in X^{*}:\left\langle x^{*}, x\right\rangle=0, \forall x \in L\right\}$. Thus

$$
\begin{aligned}
\left(x^{*}, 0\right) & \in \bigcap_{L \in \mathscr{F}(X)}\left[\{0\} \times B_{Y}^{*}+3\left(k_{F}+1\right) \partial_{A} d\left(x_{0}, y_{0} ; \mathrm{Gr} F\right)+L^{\perp} \times\{0\}\right] \\
& =\{0\} \times B_{Y}^{*}+k \partial_{A} d\left(x_{0}, y_{0} ; \mathrm{Gr} F\right)
\end{aligned}
$$

where $k=3\left(k_{F}+1\right)$.

Now we are in a position to state our main result of this section concerning Lagrange multipliers of Kuhn-Tucker type for (P).

Theorem 5.3. Suppose $F$ is pseudo-Lipschitz at $\left(x_{0}, 0\right)$ and the multivalued mapping

$$
F^{C}(x)= \begin{cases}F(x) & \text { if } x \in C, \\ +\varnothing & \text { otherwise }\end{cases}
$$

is metrically regular at $\left(x_{0}, 0\right)$. If $x_{0} \in C \cap F^{-1}(0)$ is a weak Pareto local minimum for $(\mathrm{P})$, there exist $k>0, y^{*} \in Y^{*}$, and $z^{*} \in Z^{*} \backslash\{0\}$ such that

$$
\left\langle z^{*}, z\right\rangle \geq 0 \text { for all } z \in P
$$

and

$$
0 \in \partial_{A}\left(z^{*} \circ f\right)\left(x_{0}\right)+D^{*} F\left(x_{0}, 0\right)\left(y^{*}\right)+k \partial_{A} d\left(x_{0}, C\right) .
$$

Proof. First note that $C \cap F^{-1}(0)=\left(F^{C}\right)^{-1}(0)$. By Proposition 2.4.3 in Clarke [7] and Lemma 5.1, $x_{0}$ is a local minimum of the function

$$
x \rightarrow p(f(x)-b)+\gamma d\left(x,\left(F^{C}\right)^{-1}(0)\right)
$$

for some $b \in f(x)-\operatorname{int} P$ and $\gamma>0$. Using the metric regularity of $F^{C}$ we get that the function

$$
h(x)=p(f(x)-b)+a \gamma(d(0, F(x))+d(x, C))
$$

attains a local minimum at $x_{0}$ for some $a>0$. Then $0 \in \partial_{A} h\left(x_{0}\right)$ and hence, by Theorem 2.2 and the subdifferential formula of a sum in Ioffe [16-17], there exists $z^{*} \in \partial_{A} p\left(f\left(x_{0}\right)-b\right)$ such that

$$
0 \in \partial_{A}\left(z^{*} \circ f\right)\left(x_{0}\right)+a \gamma \partial_{A} d(0, F(\cdot))\left(x_{0}\right)+a \gamma \partial_{A} d\left(x_{0}, C\right) \text {. }
$$

Thus, by Lemma 5.2, there exists $y^{*} \in Y^{*}$ satisfying

$0 \in \partial_{A}\left(z^{*} \circ f\right)\left(x_{0}\right)+\left\{x^{*} \in X^{*}:\left(x^{*},-y^{*}\right) \in k \partial_{A} d\left(x_{0}, 0 ; \mathrm{Gr} F\right)\right\}+k \partial_{A} d\left(x_{0}, C\right)$

for some $k>a \gamma$. 
The theorem will therefore be proved if we show that $z^{*} \neq 0$ and $\left\langle z^{*}, z\right\rangle \geq$ 0 , for all $z \in P$. Indeed, since $p$ is convex, we have for all $z \in Z$

$$
\left\langle z^{*}, z-f\left(x_{0}\right)+b\right\rangle \leq p(z)-p\left(f\left(x_{0}\right)-b\right) .
$$

On the one hand we have

$$
\left\langle z^{*}, f\left(x_{0}\right)-b\right\rangle \geq p\left(f\left(x_{0}\right)-b\right)=1
$$

and so $z^{*} \neq 0$. On the other hand, fix $z \in P$. As $f\left(x_{0}\right)-b \in \operatorname{int} P$ there exists $t>0$ satisfying $f\left(x_{0}\right)-b-t z \in$ int $P$. So $f\left(x_{0}\right)-b-t z \in P$ and $f\left(x_{0}\right)-b \in f\left(x_{0}\right)-b-t z+P$ and hence by increasing monotonicity of $p$ over $P$ and by (9) we get

$$
\left\langle z^{*},-t z\right\rangle \leq p\left(f\left(x_{0}\right)-b-t z\right)-p\left(f\left(x_{0}\right)-b\right) \leq 0 .
$$

Therefore $\left\langle z^{*}, z\right\rangle \geq 0$ and the proof is complete.

By Theorem 5.3 and Corollary 4.5 we have

Corollary 5.4. Suppose $F$ is pseudo-Lipschitz and uniformly compactly epiLipschitz at $\left(x_{0}, 0\right)$ and $x_{0} \in C \cap F^{-1}(0)$ is a weak Pareto local minimum for $(\mathrm{P})$. Suppose also that for all nonzero $y^{*} \in Y^{*}$

$$
0 \notin D^{*} F\left(x_{0}, 0\right)\left(y^{*}\right)+\mathbb{R}_{+} \partial_{A} d\left(x_{0}, C\right) .
$$

Then the conclusion of Theorem 5.3 holds.

As a consequence we have the following result which concerns the Lagrange multipliers of Fritz-John type for $(\mathbf{P})$.

Theorem 5.5. Suppose $F$ is pseudo-Lipschitz and uniformly compactly epiLipschitz at $\left(x_{0}, 0\right)$ and $x_{0} \in C \cap F^{-1}(0)$ is a weak Pareto local minimum for $(\mathrm{P})$. Then there are $k>0, y^{*} \in Y^{*}$, and $z^{*} \in Z^{*}$ such that

$$
\left(z^{*}, y^{*}\right) \neq(0,0), \quad\left\langle z^{*}, z\right\rangle \geq 0, \quad \text { for all } z \in P
$$

and

$$
0 \in \partial_{A}\left(z^{*} \circ f\right)\left(x_{0}\right)+D^{*} F\left(x_{0}, 0\right)\left(y^{*}\right)+k \partial_{A} d\left(x_{0}, C\right) .
$$

Proof. Let $F^{C}$ be as in Theorem 5.3. If $F^{C}$ is metrically regular at $\left(x_{0}, 0\right)$, then Theorem 5.3 gives us the desired result. If it is not the case, the assumption of Corollary 4.5 is not satisfied and so there exists a nonzero $y^{*} \in Y^{*}$ such that

$$
0 \in D^{*} F\left(x_{0}, 0\right)\left(y^{*}\right)+\mathbb{R}_{+} \partial_{A} d\left(x_{0}, C\right) .
$$

Thus it suffices to take $z^{*}=0$ and the proof is complete.

As a particular case of problem $(\mathrm{P})$ we consider the following Pareto mathematical programming problem

$$
\begin{array}{ll}
\operatorname{minimize} & f(x) \\
\text { subject to } & x \in C, \\
& g(x) \in D
\end{array}
$$

where $g: X \rightarrow Y$ is a strongly compactly Lipschitzian mapping at $x_{0}$ and $D$ is a closed subset of $Y$ which is compactly epi-Lipschitz at $g\left(x_{0}\right) \in D$. 
Corollary 5.6. Suppose $x_{0} \in C \cap F^{-1}(0)$ is a weak Pareto local minimum for $\left(\mathbf{P}^{\prime}\right)$. Then there are $k>0, y^{*} \in k \partial_{A} d\left(g\left(x_{0}\right), D\right)$, and $z^{*} \in Z^{*}$ such that

$$
\left(z^{*}, y^{*}\right) \neq(0,0), \quad\left\langle z^{*}, z\right\rangle \geq 0 \text { for all } z \in P
$$

and

$$
0 \in \partial_{A}\left(z^{*} \circ f\right)\left(x_{0}\right)+\partial_{A}\left(y^{*} \circ g\right)\left(x_{0}\right)+k \partial_{A} d\left(x_{0}, C\right) .
$$

Proof. It suffices to observe, via Theorem 2.2, that for $F(x)=-g(x)+D$

$$
\partial_{A} d\left(0, F\left(x_{0}\right)\right) \subset \bigcup_{y^{*} \in \partial_{A} d\left(g\left(x_{0}\right), D\right)}\left[\partial_{A}\left(y^{*} \circ g\right)\left(x_{0}\right) \times\left\{y^{*}\right\}\right]
$$

and Theorem 5.5 completes the proof.

Remark. Note that our corollary speaks to the following situations:

- $D$ is epi-Lipschitz in the sense of Rockafellar [37] and so in particular when $D$ is a closed convex cone with nonempty interior, or

- $D=D_{1} \times\{0\}$ with $D_{1}$ compactly epi-Lipschitz and $\{0\} \subset \mathbb{R}^{n}$.

After we completed this work, we received the paper [32] by Mordukhovich and Shao which treats characterizations of the metric regularity of multivalued mappings between Banach spaces admitting equivalent Fréchet differentiable norms.

\section{REFERENCES}

1. J. P. Aubin, Lipschitz behavior of solutions to nonconvex problems, Math. Oper. Res. 9 (1984), 87-111.

2. J. P. Aubin and H. Frankowska, On inverse functions theorems for set-valued maps, J. Math. Pures Appl. (9) 71 (1987), 71-89.

3. J. M. Borwein, Stability and regular point of inequality systems, J. Optim. Theory Appl. 48 (1986), 9-52.

4. __ Epi-Lipschitz-like sets in Banach space: theorems and examples, Nonlinear Anal. Theory Methods Appl. 11 (1987), 1207-1217.

5. J. M. Borwein and H. M. Strojwas, Tangential approximations, Nonlinear Anal. Theory Methods Appl. 9 (1985), 1347-1366.

6. J. M. Borwein and D. M. Zhuang, Verifiable necessary and sufficient conditions for openness and regularity for set-valued maps, J. Math. Anal. Appl. 47 (1988), 441-459.

7. F. H. Clarke, Optimization and nonsmooth analysis, Wiley-Interscience, New York, 1983.

8. S. Dolecki, Semi continuity in constrained optimization. I, Control Cybernet. 7 (1978), 5-16.

9. $ـ$ Semi continuity in constrained optimization. Ib, Control Cybernet. 7 (1978), 17-28.

10. __ Semi continuity in constrained optimization, II, Control Cybernet. 7 (1978), 51-68.

11. I. Ekeland, On the variational principle, J. Math. Anal. Appl. 47 (1974), 324-353.

12. H. Frankowska, An open mapping principle for set-valued maps, J. Math. Anal. Appl. 127 (1987), 172-180.

13. L. M. Graves, Some mapping theorems, Duke Math. J. 17 (1950), 111-114.

14. A. D. Ioffe, Regular points of Lipschitz mappings, Trans. Amer. Math. Soc. 251 (1979), 61-69.

15. __ Approximate subdifferentials and applications 1: The finite dimensional theory, Trans. Amer. Math. Soc. 281 (1984), 289-316.

16. _ـ Approximate subdifferentials and applications 2: Functions on locally convex spaces, Mathematika 33 (1986), 111-128.

17. _ Approximate subdifferentials and applications 3: Metric theory, Mathematika 36 (1989), 1-38. 
18. 203-218.

19. A. Jourani, Regularity and strong sufficient optimality conditions in differentiable optimization problems, Numerical Funct. Anal. Optim. 14 (1993), 69-87.

20. problems under inclusion constraints, J. Optim. Theory Appl. 81 (1994), 97-120.

21. __ Qualification conditions for multivalued functions in Banach spaces with applications to nonsmooth vector optimization problems, Math. Programming 66 (1994), 1-23.

22. A. Jourani and L. Thibault, Approximate subdifferential and metric regularity: The finite dimensional case, Math. Programming 47 (1990), 203-218.

23. Approximations and metric regularity in mathematical programming in Banach space, Math. Oper. Res. 18 (1993), 390-401.

24. __ Metric regularity for strongly compactly Lipschitzian mappings (to appear).

25. _ Approximate subdifferentials of composite functions, Bull. Austral. Math. Soc. $\mathbf{4 7}$ (1993), 443-455.

26. A. Ya. Kruger, A covering theorem for set-valued mappings, Optim. 19 (1988), 763-780.

27. L. Ljusternik, Conditional extrema of functional, Math. USSR-Sb. 41 (1934), 390-401.

28. P. D. Loewen, Limits of Fréchet normals in nonsmooth analysis, Optimization and Nonlinear Analysis (A. Ioffe, M. Marcus, and S. Reich, eds.) Pitman Res. Notes Math., 1990, pp. $178-188$.

29. B. S. Mordukhovich, Nonsmooth analysis with nonconvex generalized differentials and adjoint mappings, Dokl. Akad. Nauk UzSSR 28 (1984), 976-979.

30. _ Approximation methods in problems of optimization and control, "Nauka", Moscow, 1988; English transl., Wiley-Interscience (to appear) .

31. Complete characterization of openness, metric regularity, and Lipschitzian properties of multifunctions, Trans. Amer. Math. Soc. (to appear).

32. B. S. Mordukhovich and Y. Shao, Differential characterizations of covering and metric regularity of multifunction between Banach spaces (to appear).

33. J. P. Penot, Metric regularity, openness and Lipschitzian behavior of multifunctions, Nonlinear Anal. Theory Methods Appl. 13 (1989), 629-643.

34. S. M. Robinson, Regularity and stability for convex multivalued functions, Math. Oper. Res. 1 (1976), 130-143.

35. __ Stability theorems for systems of inequalities, Part II: differential nonlinear systems, SIAM J. Numerical Anal. 13 (1976), 497-513.

36. R. T. Rockafellar, Directionally Lipschitzian functions and subdifferential calculus, Proc. London Math. Soc. (3) 39 (1978), 331-355.

37. (1985), 867-885.

38. L. Thibault, Subdifferentials of compactly Lipschitzian vector-valued functions, Travaux du Séminaire d'Analyse Convexe, vol. 8, U.E.R. Math., Montpellier, 1978.

39. __ Lagrange-Kuhn-Tucker multipliers for Pareto optimization problems, Optimization and nonlinear analysis (A. Ioffe, M. Marcus, and S. Reich, eds.), Pitman Res. Notes Math. Ser., Longman Sci. Tech., Harlow, 1990.

40. __ Lagrange-Kuhn-Tucker multipliers for Pareto optimization problems (to appear).

41. _ On subdifferentials of optimal value functions, SIAM J. Control Optim. 29 (1991), 1019-1036.

42. C. Ursescu, Multifunctions with convex closed graph, Czechoslovak Math. J. 25 (1975), 438-441.

Université de Bourgogne, Laboratoire d'Analyse Numérique, B. P. 138, 21004, Dijon CEDEX, FRANCE

Université Montpellier II, Department des Sciences, Mathématiques, 34095-MontPellier Cedex 5, France 